\title{
Nachhaltige Entwicklung zurück auf der Tagesordnung
}

Eine unbequeme Wahrheit ist angekommen: Die Berichte des UN-Klimarats (IPCC) zu den Folgen des Klimawandels waren ein Weckruf für Politik und Bevölkerung. Mittlerweile sind zwei Drittel der Ansicht, dass Deutschland in der internationalen Klimaschutzpolitik vorangehen sollte, so die Studie „Umweltbewusstsein 2006 “ des Umweltbundesamts. Vor zwei Jahren waren es noch gut $10 \%$ weniger. Auch Bundeskanzlerin Angela Merkel hat das Gelingen des G8-Gipfels von einem Beschluss zum Klimaschutz abhängig gemacht. Doch was passiert, wenn wir jetzt einen normalen Sommer erleben und der nächste Winter Schnee und Eis bringt, ganz wie gehabt? Bleibt das Thema dann auf der Tagesordnung? Und: Sind die Ansätze der Regierung ausreichend? Stimmt die Rhetorik - vor allem auf internationaler Bühne - mit dem Handeln auf bundespolitischer Ebene überein?

Das Konzept einer nachhaltigen Entwicklung bietet die Chance, langfristige Lösungen zu formulieren und Kernthemen miteinander $z u$ verbinden. Eine nach haltige Entwicklung hat zum Ziel, unsere Lebensgrundlagen zu erhalten, im Norden wie im Süden, sowohl jetzt als auch für die nachfolgenden Generationen. Das Weltklima ist dabei ein entscheidender Parameter.

Das Instrument der Bundesregierung dafür ist die Nationale Nachhaltigkeitsstrategie aus dem Jahr 2002. Ihr Kernstück bildet das Set der 21 langfristigen und quantitativen Ziele und Indikatoren. Sie sollen die Richtung weisen - ̈̈ber diese und die nächste Legislaturperiode hinaus. Darunter sind einige anspruchsvolle Umweltziele, wie der Ausbau des Ökolandbaus auf $20 \%$ der landwirtschaftlich genutzten Fläche bis 2010 und die Verringerung des Flächenverbrauchs auf 30 Hektar am Tag bis zum Jahr 2020. Das Klimaschutzziel hingegen reicht nicht weit genug: Wie bereits im Kyoto-Protokoll vereinbart, sollen die Treibhausgas-Emissionen bis zum Zeitraum 2008 bis 2012 um $21 \%$ gegenüber 1990 reduziert werden. Für die erneuerbaren Energien enthält die Strategie das Ziel, bis zum Jahr 2010 den Anteil an der Stromerzeugung auf 12,5\% zu erhöhen. Hier hat die Bundesregierung mittlerweile ein ehrgeizigeres Vorhaben verkündet: $27 \%$ will sie bis 2020 erreichen.

Zum Stand der Umsetzung der Nachhaltigkeitsziele hat die Bundesregierung im April dieses Jahres einen Bericht des Statistischen Bundesamts veröffentlicht. Sie kommt damit der zweijährigen Berichtspflicht nach. Doch der sogenannte Indikatorenbericht zeigt deutlich: Das Gros der Ziele ist von einer Umsetzung weit entfernt. So betrug der Anteil des Ökolandbaus 2005 nur 4,7 \% und die Inanspruchnahme neuer Flächen immer noch 118 Hektar am Tag. Wenn die Regierung ihr Tempo nicht erhöht, droht die Nachhaltigkeitsstrategie zu scheitern.

Die Umweltverbände schätzen zudem kritisch ein, dass die Nachhaltigkeitsstrategie in zentralen Bereichen allein auf relative Effizienzziele und nicht auf absolute Umweltentlastungen setzt. Dass solche
Effizienzziele zu kurz greifen, zeigen nun auch die aktuellen Daten des Statistischen Bundesamts. So reicht z. B. das Ziel der Steigerung der Energieproduktivität nicht aus. Hier wird die Verringerung des Energieverbrauchs ins Verhältnis gestellt zum - steigenden - Bruttosozialprodukt. Notwendig ist es aber, den realen Energieverbrauch zu senken: Dieser ging trotz einer Produktivitätssteigerung von etwa $30 \%$ in den letzten 15 Jahren um nur $3 \%$ zurück. Insgesamt muss es darum gehen, eine absolute Reduktion im Umweltverbrauch zu erreichen, da die natürlichen und nicht erneuerbaren Ressourcen und die Aufnahmekapazität der Erde für Schadstoffe begrenzt sind.

Die Folgen der Erderwärmung sind nicht rückholbar, und es ist allerhöchste Zeit, vorzusorgen, absolute Zielmarken zu setzen und konsequent zu handeln. Maximal zwei Grad Erderwärmung sind tragbar. Um diese nicht zu überschreiten, müssen die Industriestaaten, die historisch und aktuell die Hauptverursacher des Klimawandels sind, ihren $\mathrm{CO}_{2}$-Ausstoß bis 2050 um $80 \%$ reduzieren. Ein Ziel, das in der Nachhaltigkeitsstrategie bislang fehlt. Deutschland und die EU müssen hier eine verbindliche Position einnehmen und auf der Klimakonferenz im Dezember 2007 in Bali eine entsprechende internationale Vereinbarung erreichen.

Der BUND hat mit seiner Studie „Zukunftsfähiges Deutschland“ bereits im Jahr 1995 einen wichtigen Impuls für die Nachhaltigkeitsdiskussion gegeben. Die Studie basiert auf dem Konzept des „Umweltraums". Die Tragfähigkeit der Ökosysteme wie unseres Klimas, die Verfügbarkeit von Rohstoffen und die Regenerationsfähigkeit natürlicher Ressourcen sind begrenzt. Und in diesem begrenzten Umweltraum haben alle Menschen in gleicher Weise Nutzungsrechte. Nun, zwölf Jahre später, plant der BUND gemeinsam mit „Brot für die Welt" und dem Evangelischen Entwicklungsdienst (EED) eine Studie „Zukunftsfähiges Deutschland in einer globalisierten Welt“. Denn: Die Globalisierung schafft eine veränderte Ausgangssituation und neue Herausforderungen. Die soziale Situation in Deutschland hat sich zugespitzt. Die physischen Grenzen sind klarer geworden, sei es beim Klima oder bei den Erdölressourcen. Die Verbindung mit Fragen globaler Gerechtigkeit und Friedenssicherung ist brisanter geworden. Offen ist, ob Nachhaltigkeitsstrategien mit einem weiterhin angestrebten Wirtschaftswachstum vereinbar sind. Im Zentrum der Untersuchung wird es daher darum gehen, wie sich wirtschaftliche Entwicklung mit einem ressourcenleichten Wohlstand verbinden lässt. Diese Frage stellt sich nicht nur für uns. Sie hat weltweite Relevanz. Das betrifft auch die aufstrebenden Ökonomien Indiens und Chinas.

Dr. Angelika Zahrnt, Vorsitzende des BUND (Bund für Umwelt und Natur Deutschland e. V.) und Mitglied im Rat für Nachhaltige Entwicklung. e-mail: angelika.zahrnt@bund.net 